

Revista cientifica de investigación actualización del mundo de las ciencias

Ronald Alfredo Cevallos Macías a, Diana Katherine Suárez Intriago b; Silvia Karina Briones Valencia ${ }^{c}$, Evelyn Estefanía Calderón López ${ }^{\text {d; }}$

Marvin Damián Veliz Mero ${ }^{\mathrm{e}}$; Marcos Andrés Campozano Burgos ${ }^{\mathrm{f}}$

Tratamiento de parasitosis intestinal en niños menores de 5 años

Tratamiento de parasitosis intestinal en niños menores de 5 años

Revista Científica de Investigación actualización del mundo de las Ciencias. Vol. 3 núm., 1, enero, ISSN: 2588-0748, 2018, pp. 722-749

DOI: $10.26820 /$ reciamuc/3.(1).enero.2019.722-749

URL: http://reciamuc.com/index.php/RECIAMUC/article/view/255

Código UNESCO: 3205 Medicina Interna

Tipo de Investigación: Artículo de Revisión

Editorial Saberes del Conocimiento

Recibido: 10/12/2018

Aceptado: 20/01/2019

Publicado: 30/01/2019

Correspondencia: rocemac@hotmail.com

a. Médico Cirujano; rocemac@ @otmail.com

b. Médico Cirujano; dksii_45@ hotmail.com

c. Médico Cirujano; silkabriones16@hotmail.com

d. Médico Cirujano; ivitef89@gmail.com

e. Médico Cirujano; drmarveliz@ hotmail.com

f. Médico Cirujano; andres_bigman25@hotmail.com 


\section{Tratamiento de parasitosis intestinal en niños menores de 5 años}

Vol. 3, núm. 1., (2019)

Ronald Alfredo Cevallos Macías; Diana Katherine Suárez Intriago; Silvia Karina Briones Valencia, Evelyn Estefanía Calderón López; Marvin Damián Veliz Mero; Marcos Andrés

Campozano Burgos

\section{RESUMEN}

Las infecciones parasitarias intestinales provocan un número importante de infestaciones en niños en países latinoamericanos Los parásitos más frecuentes son: giardias, oxiuros y, en menor grado, áscaris. En los niños menores de cinco años es frecuente encontrar sintomatología digestiva, de la cual la más frecuente es la diarrea. Las causas pueden ser múltiples y, aunque las más frecuentes son bacterias y virus, los parásitos también pueden ser causa de diarrea, especialmente los protozoos (Entamoeba histolytica y Giardia lamblia); por lo que, la determinación de parásitos en tres muestras de heces seriadas estará indicada siempre en la primera visita en estos pacientes y siempre que haya sintomatología sugestiva, lo que constituye un gran problema de salud pública debido a los altos índices generados por su incidencia y los altos costos que implica para los sistemas de salud su alta demanda y las atenciones ambulatorias y hospitalizaciones generadas a causa de la misma. Entre los factores determinantes que originan estas infecciones se encuentran las condiciones geográficas, sociales, culturales y de servicios públicos; lo que ha llevado a que la misma constituya una de las consultas más frecuentes en los servicios de consulta externa, urgencias y consultorios de médicos generales y pediatras. Para su tratamiento básico se recomienda, cumplir con las medidas sanitarias indicadas, las mismas deben iniciarse mediante el consumo de aguas potables o debidamente hervidas, de buenas condiciones higiénicas, alimentarias y adecuado control de las excretas; ya que su propagación se realiza por vía fecal oral. Este tipo de infecciones gástricas de manera general se encuentra determinado por diferentes causas, incluyéndose entre ellas, escasa formación socio - cultural, bajo nivel socio económico y deficiente información sobre las medidas higiénico - sanitarias.

Palabras Claves: Parasitosis Intestinal; Infecciones Gástricas; Diarreas; Giardias; Oxiuros; Áscarasis; Protozoos; Problema de Salud Pública. 


\title{
Tratamiento de parasitosis intestinal en niños menores de 5 años
}

Vol. 3, núm. 1., (2019)

Ronald Alfredo Cevallos Macías; Diana Katherine Suárez Intriago; Silvia Karina Briones

Valencia, Evelyn Estefanía Calderón López; Marvin Damián Veliz Mero; Marcos Andrés

Campozano Burgos

\begin{abstract}
Intestinal parasitic infections cause a significant number of infestations in children in Latin American countries. The most frequent parasites are: giardias, pinworms and, to a lesser degree, ascarids. In children under five years, it is common to find digestive symptoms, of which the most frequent is diarrhea. The causes can be multiple and, although the most frequent are bacteria and viruses, parasites can also cause diarrhea, especially protozoa (Entamoeba histolytica and Giardia lamblia); therefore, the determination of parasites in three stool samples will always be indicated at the first visit in these patients and whenever there are suggestive symptoms, which constitutes a major public health problem due to the high rates generated by their incidence and the high costs implied by health systems for their high demand and ambulatory care and hospitalizations generated because of it. Among the determining factors that originate these infections are the geographical, social, cultural and public service conditions; which has led to it being one of the most frequent consultations in the services of external consultation, emergencies and offices of general practitioners and pediatricians. For its basic treatment, it is recommended to comply with the sanitary measures indicated, they should be initiated by drinking potable water or properly boiled, with good hygienic, food and adequate control of the excreta; since its spread is done by fecal - oral route. This type of gastric infections in general is determined by different causes, including among them, poor socio - cultural formation, low socio - economic level and poor information on hygienic - sanitary measures.
\end{abstract}

Key Words: Intestinal Parasitosis; Gastric Infections; Diarrhea; Giardias; Pinworms; Ascarasis; Protozoa; Problem Public Health. 


\section{Tratamiento de parasitosis intestinal en niños menores de 5 años}

Vol. 3, núm. 1., (2019)

Ronald Alfredo Cevallos Macías; Diana Katherine Suárez Intriago; Silvia Karina Briones Valencia, Evelyn Estefanía Calderón López; Marvin Damián Veliz Mero; Marcos Andrés

Campozano Burgos

\section{Introducción.}

A través de la historia de la humanidad, los pueblos han buscado mantener, proteger y recuperar su salud: para ello, los gobiernos han organizado diferentes políticas con el fin de optimizar los recursos humanos, físicos, económicos y ambientales, partiendo de enfoques y estrategias variadas de acuerdo al momento y situación social que se esté viviendo. De aquí, la importancia de los programas de salud comunitarios establecidos por los gobiernos y las diferentes organizaciones, los cuales buscan concientizar a los habitantes de las diferentes poblaciones sobre las causas y efectos que tienen determinadas patologías médicas con el fin de promocionar la salud.

Para (Vieira, 2011), "la actual circunstancia histórica, política, económica y social, que viven las Naciones latinoamericanas, reclaman la mejora, eficacia y eficiencia del sistema de los servicios de salud". (p.32). En tal sentido, es importante entender que entre los propósitos que encierran estos programas o medidas médicas de prevención, se encuentra educar e influir en el estilo de vida de los ciudadanos, con el fin de minimizar las enfermedades de alta prevalencia, como lo es la parasitosis intestinal, por ser ésta desde una perspectiva mundial un problema de salud pública importante; en especial en países subdesarrollados en donde sus consecuencias originan altos índices de morbilidad y mortalidad. Para (Blanco, 2011):

La mortalidad que origina en el mundo las tres infecciones parasitarias intestinales más frecuentes no es nada despreciable: obsérvese que 60.000 muertes por año para $A$. lumbricoides, 65.000 muertes por año para A. doudenale y N. americanus y 10.000 muertes por año para T. trichiura. (p.65).

Lo descrito permite señalar que, las enfermedades intestinales causadas por helmintos afectan cerca de un tercio de la población mundial, sobre todo a aquellas personas que habitan en países en vías de desarrollo, particularmente en aquellos de clima tropical, los cuales tienen la tendencia a ser los más afectados, agregándose las adversas condiciones sociales y económicas en las que se desenvuelven. 


\section{Tratamiento de parasitosis intestinal en niños menores de 5 años}

Vol. 3, núm. 1., (2019)

Ronald Alfredo Cevallos Macías; Diana Katherine Suárez Intriago; Silvia Karina Briones Valencia, Evelyn Estefanía Calderón López; Marvin Damián Veliz Mero; Marcos Andrés Campozano Burgos

De igual forma hay que señalar que, las enfermedades parasitarias, sobre todo aquellas de transmisión hídricas (entre ellas la parasitosis intestinal) son la segunda causa de ingresos en los Hospitales Públicos Latinoamericanos. De ahí, que se observen cálculos aproximados de la frecuencia de infecciones parasitarias en el mundo, las cuales afectan de mayor forma a niños menores de cinco años, generando como respuesta cifras superiores a cuatro millones de personas infectadas. En apoyo a este planteamiento, (Vianco, 2011), expone:

Las parasitosis intestinales constituyen en países latinoamericanos indicadores sensibles de los factores ecológicos, y en particular, de aquellos derivados del ambiente natural o de las modificaciones introducidas por el hombre (industrias, represas, carreteras, basurales, cultivos agrícolas y proyectos pecuarios, deforestación, contaminación de aguas, suelos y atmósfera. (p.32).

Tal señalamiento lleva a destacar que, lo mismo ha sucedido con las entero parasitosis en Latinoamérica, en donde el agente causal de la blastocistosis, el Blastocystis hominis viene destacando, aún, cuando persisten aspectos controversiales sobre el mismo, como por ejemplo su rol patógeno. Además de la inmunosupresión, se observa que estos países comprenden diversos fenómenos sociológicos, los cuales hacen que el tema sobre esta enfermedad tenga interés en la actualidad; es por ello que, la orientación del problema en estos casos debe ser abordada, teniendo en cuenta no sólo la sintomatología sino el origen de la población o el lugar de desplazamiento ya que en ella predominarán unos determinados patógenos sobre otros. Considerando lo descrito, (Rossiet, 2011):

Las enfermedades parasitarias son responsables de una morbilidad considerable en el mundo entero, afectando de manera especial a niños; las mismas se presentan frecuentemente con síntomas no específicos y altas tasas de prevalencia. Las ascariosis, tricocefalosis, giardiosis y amebiosis se encuentran entre las diez infecciones más comunes observadas en el mundo. (p.32). 


\section{Tratamiento de parasitosis intestinal en niños menores de 5 años}

Vol. 3, núm. 1., (2019)

Ronald Alfredo Cevallos Macías; Diana Katherine Suárez Intriago; Silvia Karina Briones Valencia, Evelyn Estefanía Calderón López; Marvin Damián Veliz Mero; Marcos Andrés

Campozano Burgos

Lo anteriormente expuesto, permite resaltar que la parasitosis se ha convertido en una enfermedad que hay que atacar, ya que su prevalencia depende en gran medida de los hábitos higiénicos de la población, y se encuentra relacionada con conductas socioeconómicas y culturales; problemática ésta que, lleva a considerar la necesidad de ejecutar planes de intervención comunitarios, los cuales les permitan a las comunidades tomar las previsiones médicas necesarias para disminuir los riesgos de contagios de este tipo de patología; pues la misma es originada, en muchos de los casos por ingestión de alimentos contaminados, inadecuados hábitos higiénicos, condiciones ambientales inapropiadas, bajo nivel socio - económico, entre otras tantas causantes.

En la misma línea de ideas, se debe expresar que el parasitismo intestinal es una enfermedad que afecta a todas las personas por igual, sin embargo la población infantil por sus características poseen un nivel de susceptibilidad elevado de padecerlas, ya que existe mayor oportunidad de contacto con dichos parásitos porque permanecen gran parte del día en las escuelas y desarrollan actividades en colectivo, lo que puede favorecer las condiciones para la transmisión de algunas enfermedades parasitarias, especialmente aquellas en que su principal mecanismo de transmisión es la vía fecal oral, además de presentar un menor nivel inmunológico.

Significa entonces que, las infecciones parasitarias intestinales pueden afectar a todas las personas por igual, sin embargo, en la población existen diversos grupos que por sus características poseen un nivel de susceptibilidad más elevado de padecerlas, tal es el caso de los niños; en particular los mayores de 5 años de edad, porque permanecen gran parte del día en las escuelas y desarrollan actividades en colectivo, lo que puede favorecer las condiciones para la transmisión de algunas de estas patologías, especialmente aquellas en que su principal mecanismo de transmisión es la vía fecal - oral, por tal motivo se hace común que estas infecciones sean más intensas, generando en ellos, tanto efectos deletéreos sobre el crecimiento y desarrollo, como sobre el aprendizaje.

Destáquese a través de este señalamiento que, que entre las personas más afectadas con el parasitismo intestinal, se encuentran los escolares provenientes de áreas rurales; pues se observa que los mismos, además de vivir en precarias condiciones, mantienen un bajo nivel 


\section{Tratamiento de parasitosis intestinal en niños menores de 5 años}

Vol. 3, núm. 1., (2019)

Ronald Alfredo Cevallos Macías; Diana Katherine Suárez Intriago; Silvia Karina Briones

Valencia, Evelyn Estefanía Calderón López; Marvin Damián Veliz Mero; Marcos Andrés Campozano Burgos

socioeconómico, lo que en consecuencia genera la falta de educación sanitaria tanto en los niños como en sus grupos familiares. Estos factores conducen a que un grupo considerable de estas familias mantengan un desconocimiento total acerca de la parasitosis intestinal, lo que se hace evidente en el hecho de que en cada núcleo familiar exista una carencia general e inmediata de las más elementales medidas higiénicas, las cuales se deben tener en cuenta a la hora de preparar y distribuir los alimentos.

En virtud de estos señalamientos, se hace referencia a la prevención como tema ineludible y forma más eficaz de controlar la aparición de un mayor número de casos de parasitismo intestinal, esta fase educativa, impone la realización de una labor de concientización de factores de riesgo sistemática que involucre a la población más afectada; claro está, teniendo en cuenta que el comportamiento humano tiene gran importancia en la transmisión de estas infecciones intestinales.

Se indica con esto, que el principal tratamiento contra esta enfermedad se basa en el modo de prevención individual y colectiva, el cual surge de ejercer acciones sobre todos los factores de riesgo que influyen en la aparición del parasitismo intestinal; claro está, que para el logro de ello, se han establecido innumerables medidas tales como: ingestión de agua potable, hervida o clorada, lavado adecuado de los alimentos, verduras y frutas antes de su ingestión, consumo de carnes frescas y bien cocidas, así como leche pasteurizada o hervida, mantener a los animales alejados de los alimentos, lavado de las manos después de defecar y antes de manipular alimentos, higiene adecuada de las manos de los niños después de jugar en la tierra o en el suelo y evitar el fecalismo al aire libre.

Por consiguiente, la prevención representa una de las mejores maneras de detener la aparición y transmisión de las enfermedades parasitarias; siendo la misma lograda, a través de una permanente labor educativa, la cual puede ser llevada a cabo mediante el desarrollo de proyectos, planes comunitarios, jornadas de salud y en todos aquellos escenarios en los que se pueda impartir orientaciones sanitarias a las personas; a fin de que éstas adquieran una instrucción significativa que les permitirá una mejor calidad de vida; garantizándoles esto, a los representantes de la salud la prevención de este tipo de patologías. 


\section{Tratamiento de parasitosis intestinal en niños menores de 5 años}

Vol. 3, núm. 1., (2019)

Ronald Alfredo Cevallos Macías; Diana Katherine Suárez Intriago; Silvia Karina Briones Valencia, Evelyn Estefanía Calderón López; Marvin Damián Veliz Mero; Marcos Andrés

Campozano Burgos

Por todo esto, para lograr un intervención social significativa contra el parasitismo intestinal, es imprescindible comprender que el desarrollo de la Salud no se puede reducir a la lucha contra la enfermedad, ni a las prácticas clínicas tradicionales, sino a prevenirlas; esto, debido a que la misma, representa una de las patologías de más fácil transmisión y mayor frecuencia dentro de las poblaciones, lo cual es muy difícil de controlar, no solo por su gran difusión, sino por los diversos factores que intervienen en su cadena de propagación

\section{Tipo de Investigación.}

Dentro de toda práctica investigativa, se precisan acciones de carácter metodológico mediante las cuales, se logra conocer y proyectar los eventos posibles que la determinan, así como las características que hacen del acto científico un proceso interactivo ajustado a una realidad posible de ser interpretada. En este sentido, se puede decir, que la presente investigación corresponde al tipo documental, definido por (Dávila, 2012), "se ocupa del estudio de problemas planteados a nivel teórico, la información requerida para abordarlos se encuentra básicamente en materiales impresos, audiovisuales y /o electrónicos". (p.41).

En consideración a esta definición, la orientación metodológica permitió la oportunidad de cumplir con una serie de actividades inherentes a la revisión y lectura de diversos documentos donde se encontraron ideas explicitas relacionadas con los tópicos encargados de identificar a cada característica insertada en el estudio. Por lo tanto, se realizaron continuas interpretaciones con el claro propósito de revisar aquellas apreciaciones o investigaciones propuestas por diferentes investigadores relacionadas con el tema de interés, para luego dar la respectiva argumentación a los planteamientos, en función a las necesidades encontradas en la indagación.

\section{Fuentes Documentales.}

El análisis correspondiente a las características que predomina en el tema seleccionado, llevan a incluir diferentes fuentes documentales encargadas de darle el respectivo apoyo y en ese sentido cumplir con la valoración de los hechos a fin de generar nuevos criterios que sirven de referencia a otros procesos investigativos. Para (Arias, 2010), las fuentes documentales incorporadas en la 


\section{Tratamiento de parasitosis intestinal en niños menores de 5 años}

Vol. 3, núm. 1., (2019)

Ronald Alfredo Cevallos Macías; Diana Katherine Suárez Intriago; Silvia Karina Briones

Valencia, Evelyn Estefanía Calderón López; Marvin Damián Veliz Mero; Marcos Andrés

Campozano Burgos

investigación documental o bibliográfica, "representa la suma de materiales sistemáticos que son revisados en forma rigurosa y profunda para llegar a un análisis del fenómeno". (p.41). Por lo tanto, se procedió a cumplir con la realización de una lectura previa determinada por encontrar aquellos aspectos estrechamente vinculados con el tema sobre el "Tratamiento de Parasitosis Intestinal en niños menores de 5 años", para luego explicar mediante un desarrollo las respectivas apreciaciones generales de importancia.

\section{Técnicas para la Recolección de la Información.}

La conducción de la investigación para ser realizada en función a las particularidades que determinan a los estudios documentales, tiene como fin el desarrollo de un conjunto de acciones encargadas de llevar a la selección de técnicas estrechamente vinculadas con las características del estudio. En tal sentido, (Arias Ob cit) refiere, que es "una técnica particular para aportar ayuda a los procedimientos de selección de las ideas primarias y secundarias”. (p. 71).

Por ello, se procedió a la utilización del subrayado, resúmenes, fichaje, como parte básica para la revisión y selección de los documentos que presentan el contenido teórico. Es decir, que mediante su aplicación de estas técnicas se pudo llegar a recoger informaciones en cuanto a la revisión bibliográfica de los diversos elementos encargados de orientar el proceso de investigación. Tal como lo expresa, (Arias Ob cit) "las técnicas documentales proporcionan las herramientas esenciales y determinantes para responder a los objetivos formulados y llegar a resultados efectivos" (p. 58). Es decir, para responder con eficiencia a las necesidades investigativas, se introdujeron como técnica de recolección el método inductivo, que hizo posible llevar a cabo una valoración de los hechos de forma particular para llegar a la explicación desde una visión general.

Asimismo, se emplearon las técnicas de análisis de información para la realización de la investigación que fue ejecutada bajo la dinámica de aplicar diversos elementos encargados de determinar el camino a recorrer por el estudio, según, (Arias, Ob cit) las técnicas de procesamiento de datos en los estudios documentales "son las encargadas de ofrecer al investigador la visión o pasos que debe cumplir durante su ejercicio, cada una de ellas debe estar en correspondencia con el nivel a emplear" (p. 123). Esto indica, que para llevar a cabo el procesamiento de los datos 


\section{Tratamiento de parasitosis intestinal en niños menores de 5 años}

Vol. 3, núm. 1., (2019)

Ronald Alfredo Cevallos Macías; Diana Katherine Suárez Intriago; Silvia Karina Briones Valencia, Evelyn Estefanía Calderón López; Marvin Damián Veliz Mero; Marcos Andrés

Campozano Burgos

obtenidos, es necesario establecer las técnicas que serán seleccionadas, destacándose en este caso, de manera particular: fichas de resumen, textual, registros descriptivos entre otros, los mismos se deben ajustar al nivel que ha sido seleccionado.

\section{Resultados.}

\section{La Parasitosis Intestinal.}

Desde una perspectiva global la parasitosis intestinal, representa un problema de salud pública con mayor prevalencia en países subdesarrollados, donde provocan una importante morbimortalidad. En base a esto, se puede decir que esta enfermedad es entendida como una patología relativamente frecuente entre la población de pobreza extrema y zona rural en países en vía de desarrollo; apoyándose en esto, se señala lo descrito por el Ministerio de Salud y Desarrollo Social (MSDS) (2011), el cual indica "que la parasitosis sistemática puede ocasionar cierta morbilidad, siendo la misma un riesgo para comunidades en países del tercer mundo" (p.8). En virtud de esto, se puede decir que la parasitosis intestinal constituye una de las afecciones más frecuentes en niños y adultos del mundo en desarrollo. Su distribución es mundial y provocan trastornos que pueden ser evitados. Para (León, 2011):

Las parasitosis intestinales continúan siendo un problema de salud pública debido a que la mayoría de los infectados son asintomáticos, siendo estas más visibles cuando coexisten con etapas de mayor necesidad metabólica, de crecimiento agregándose a esto las condiciones del medio en que viven los niños, hábitos higiénicos inadecuados, entre otros, son factores, que favorecen la vía y desarrollo de la infestación parasitaria. (p.21).

Lo expuesto lleva a considerar que, las enfermedades parasitarias son responsables de una morbilidad considerable en el mundo entero; las mismas, se presentan frecuentemente con síntomas no específicos y altas tasas de prevalencia. Las ascariosis, tricocefalosis, giardiasis y amebiasis, lo que lleva a considerar que se encuentran entre las diez infecciones más comunes observadas en el mundo. De allí, que en la actualidad los autores prefieren sustituir la terminología 


\section{Tratamiento de parasitosis intestinal en niños menores de 5 años}

Vol. 3, núm. 1., (2019)

Ronald Alfredo Cevallos Macías; Diana Katherine Suárez Intriago; Silvia Karina Briones

Valencia, Evelyn Estefanía Calderón López; Marvin Damián Veliz Mero; Marcos Andrés Campozano Burgos

de parasitismo intestinal por el de enfermedades causadas por protozoarios y helmintos. En general tienen baja mortalidad, pero igualmente ocasionan importantes problemas sanitarios y sociales debido a su sintomatología y complicaciones. Por otra parte, la Organización Panamericana de la Salud (OPS, 2011), refleja que la presencia de los parásitos en niños:

Es una situación que se presenta en un $90 \%$ de los casos por falta de educación sanitaria y asistencia de control ambulatorio, ya que es más frecuente en la población rural que en la urbana debido a factores socioeconómicos, menor saneamiento ambiental y ausencia de servicios higiénicos adecuados. (p.12).

Este planteamiento, permite comprender que existen referencias que demuestran la prevalencia de altas cifras de infección, lo que lleva a considerar que deben implementarse programas de control y preservación de entero parasitosis a corto y largo plazo, así como realizar exámenes copro parasicológicos adecuados, a fin de obtener un diagnóstico óptimo y brindar un tratamiento eficaz y oportuno que permita dar de manera oportuna una mejor calidad de vida a los pobladores.

\section{Imagen $\mathbf{N}^{\circ}$ 1. Parasitosis Intestinal}

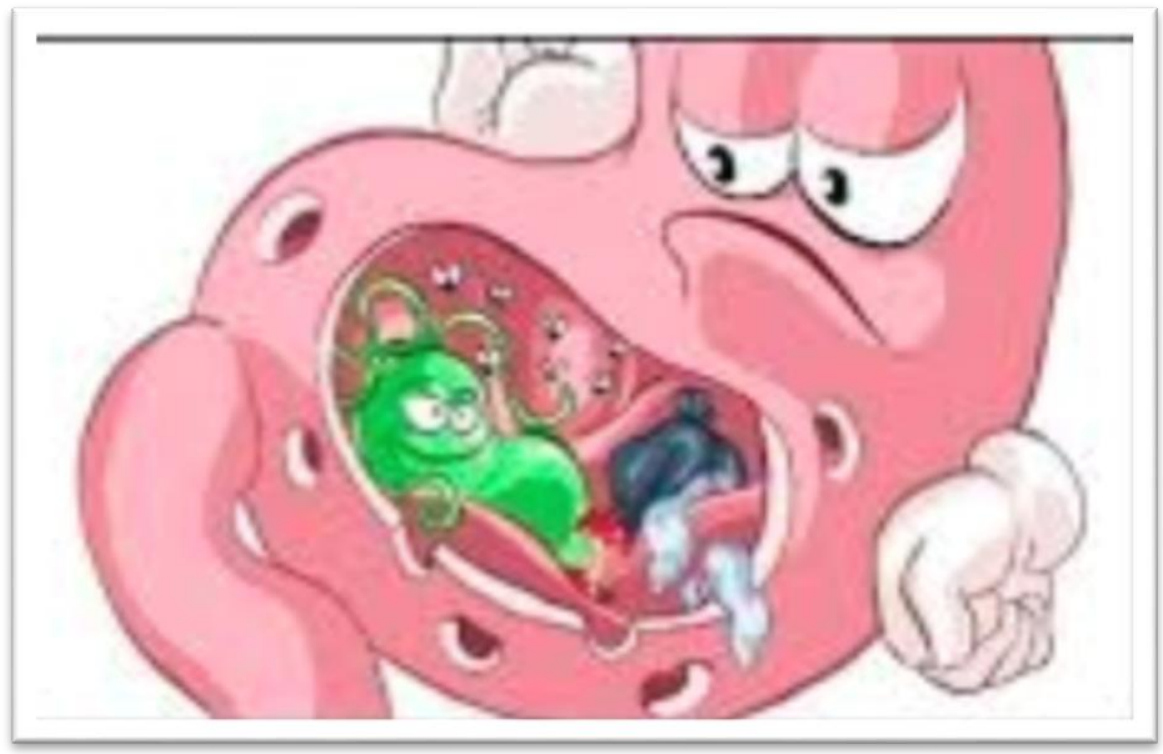

Fuente: Organización Panamericana de Salud OPS (2011) 


\section{Tratamiento de parasitosis intestinal en niños menores de 5 años}

Vol. 3, núm. 1., (2019)

Ronald Alfredo Cevallos Macías; Diana Katherine Suárez Intriago; Silvia Karina Briones Valencia, Evelyn Estefanía Calderón López; Marvin Damián Veliz Mero; Marcos Andrés

Campozano Burgos

En correspondencia a lo descrito, se destaca que la parasitosis intestinal representa un problema de alta prevalencia de tipo Blastocytis Hominis, como se observa la parasitosis es una situación que puede ser padecida por carencia de higiene y malos hábitos nutricionales, así como el uso inapropiado del calzado y falta del lavado de manos entre otros. Al respecto, (Blanco A. , 2010), establece que:

La ingestión de alimentos es el principal mecanismo de transmisión, incluso es considerada una enfermedad hídrica en los niños. El contacto directo de persona a persona parece tener mucha importancia en la transmisión. Los portadores sanos aunados al hacinamiento y las precarias condiciones de higiene determinan una mayor diseminación del flagelo" (p.3).

De acuerdo a la referencia mencionada, la parasitosis es una necesidad que hay que atacar, ya que la misma representa una enfermedad que es transmitida a través de vehículos tales como (comida, agua potable) y su prevalencia depende en gran medida de los hábitos higiénicos de la población relacionada con conductas socioeconómicas y culturales, Por todo esto, se puede decir que las infecciones y enfermedades parasitarias en el niño constituyen un importante problema de salud, el cual es más frecuente en la mayoría delos países Latinoamericanos, debido a su frecuencia, a los problemas diagnósticos y a los terapéuticos que plantean y, en ocasiones, por su gravedad. 


\section{Tratamiento de parasitosis intestinal en niños menores de 5 años}

Vol. 3, núm. 1., (2019)

Ronald Alfredo Cevallos Macías; Diana Katherine Suárez Intriago; Silvia Karina Briones

Valencia, Evelyn Estefanía Calderón López; Marvin Damián Veliz Mero; Marcos Andrés

Campozano Burgos

Tabla $\mathbf{N}^{\circ}$ 1. Prevalencia de Protozoarios por grupos de edad en niños de países Americanos

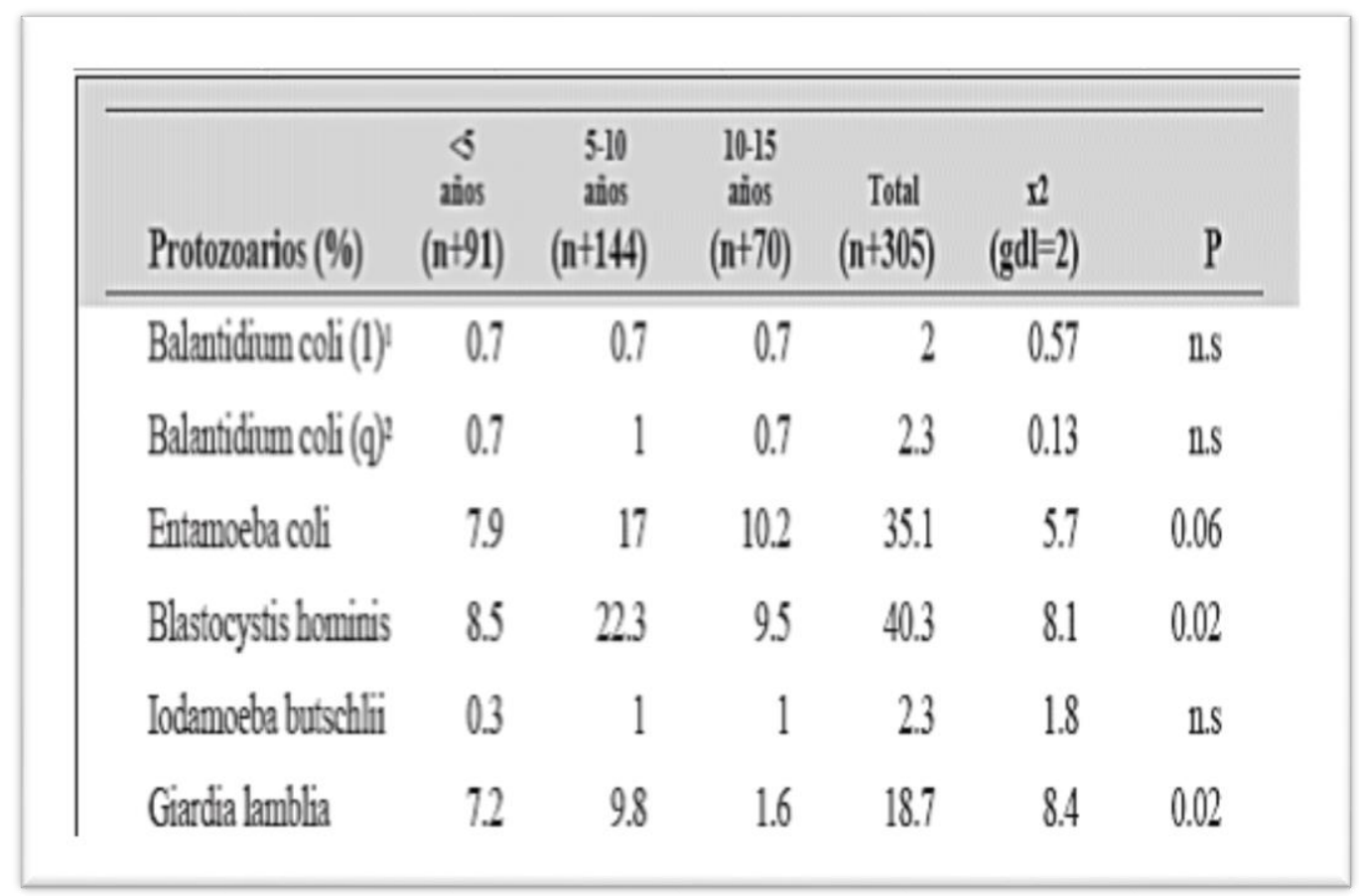

\section{Fuente: Organización Mundial de la Salud OMS (2017)}

La parasitosis es una enfermedad que repercute negativamente en el progreso socio-económico y es principal culpable de efectos sobre el estado nutricional y el estado intelectual primordialmente en los infantes, por ser éstos, los que se encuentran expuestos a mayores riesgos de contraer la enfermedad. He aquí, que se ha observado que aquellos niños poli parasitados tienen un ritmo decrecimiento inferior y su estado nutricional es deficitario afectando principalmente su desarrollo físico y mental. 
Tratamiento de parasitosis intestinal en niños menores de 5 años

Vol. 3, núm. 1., (2019)

Ronald Alfredo Cevallos Macías; Diana Katherine Suárez Intriago; Silvia Karina Briones Valencia, Evelyn Estefanía Calderón López; Marvin Damián Veliz Mero; Marcos Andrés

Campozano Burgos

Tabla $N^{\circ}$ 2. Parasitosis Intestinal según Año de Vida en niños de países Americanos

\begin{tabular}{|l|c|c|c|}
\hline \multicolumn{1}{|c|}{ Años de vida } & Estudiados & \multicolumn{2}{|c|}{ Parasitados } \\
\cline { 3 - 4 } & & No & $\%$ \\
\hline 2do & 71 & 23 & 32,4 \\
\hline 3er & 106 & 36 & 34,0 \\
\hline 4to & 105 & 57 & 54,3 \\
\hline 5to & 131 & 77 & 58,8 \\
\hline 6to & 82 & 46 & 56,1 \\
\hline Total & 495 & 239 & 48,3 \\
\hline
\end{tabular}

\section{Fuente: Organización Mundial de la Salud OMS (2017).}

Existen una serie de factores asociados al parasitismo intestinal, los cuales determinan una mejor o peor evolución de la enfermedad como son: edad, higiene personal, higiene de los alimentos, manifestaciones clínicas del parasitismo, tipo de parásito, asociaciones entre estos parásitos, tipo de lactancia recibida, nivel cultural, nivel económico, repercusión en sistema hematológico, repercusión en estado nutricional, acceso a los servicios médicos, entre otros.

En consecuencia, es de resaltar que, tanto la higiene tanto personal, como la de los alimentos y la medioambiental, es el factor de riesgo más importante en el origen y evolución del parasitismo intestinal, es decir, cuando la higiene en sus diferentes modalidades es deficiente ocurre la instalación y proliferación del parasitismo en el organismo humano, se hace persistente, crónico con los consiguientes daños en el estado nutricional e inmunológico. Destáquese a su vez que, la vía principal para la transmisión del parasitismo intestinal es la vía digestiva por el consumo de 


\section{Tratamiento de parasitosis intestinal en niños menores de 5 años}

Vol. 3, núm. 1., (2019)

Ronald Alfredo Cevallos Macías; Diana Katherine Suárez Intriago; Silvia Karina Briones

Valencia, Evelyn Estefanía Calderón López; Marvin Damián Veliz Mero; Marcos Andrés

Campozano Burgos

agua y comidas contaminadas, aunque también puede ser por el contacto directo de persona a persona.

De allí, que es importante saber que el comportamiento humano tiene gran importancia en la transmisión de las infecciones intestinales por parásitos, por lo tanto, el éxito de las medidas de control que se implementen dependerá en gran medida de la modificación que se obtenga de los hábitos en el sentido de promover la salud y no contribuir a deteriorarla, a fines de evitar que sea adquirida esta patología, caracterizada, en su efecto por algún tipo de parasito en particular.

\section{Tipos de Parasitosis.}

\section{Giardia intestinalis.}

Es un protozoo flagelado que coloniza el duodeno e intestino delgado proximal, donde puede producir una infección aguda o crónica. El ciclo vital se compone de dos estadios: trofozoítos y quistes. La vía de transmisión más frecuente es la fecal-oral. He aquí, que los niños entre 2- a 4 años tienen especial riesgo de transmisión por esta vía, principalmente los que acuden a guardería. Estos niños pueden expulsar quistes durante varios meses y las tasas de infección secundaria de familiares llegan a 5-30\%. Organización Mundial de la Salud (OMS, 2017).

Por otra parte, es importante señalar que el agua y los alimentos son también una fuente importante de transmisión. Los quistes de Giardia son viables en el agua hasta 3 meses y resisten a la cloración. La ebullición (de tan sólo un minuto) los inactiva eficazmente. También, puede transmitirse al hombre a través de animales domésticos (perros, gatos, ovejas, castores). La infección ocurre con más frecuencia en los meses de verano y otoño y es asintomática la mayoría de veces. En base a lo descrito, se debe expresar que las infecciones sintomáticas son más frecuentes en niños que en adultos; en ella, la presentación más común es una diarrea acuosa, con o sin febrícula (presente sólo en un 25\% de los pacientes), náuseas y anorexia.

Es de conocer también, que una pequeña proporción de casos evolucionan a una diarrea intermitente o más prolongada, explosiva y fétida, sin sangre, con flatulencia, dolor abdominal, anorexia y náuseas. El comienzo de la misma, puede ser agudo o gradual. La diarrea puede ser 


\section{Tratamiento de parasitosis intestinal en niños menores de 5 años}

Vol. 3, núm. 1., (2019)

Ronald Alfredo Cevallos Macías; Diana Katherine Suárez Intriago; Silvia Karina Briones Valencia, Evelyn Estefanía Calderón López; Marvin Damián Veliz Mero; Marcos Andrés

Campozano Burgos

auto limitada o crónica, produciendo en el último caso malnutrición e intolerancia a la lactosa. No es raro que sea intermitente, alternando con fases de estreñimiento; por ello, en inmuno deprimidos, la enfermedad es siempre sintomática y grave, con diarreas prolongadas y síndromes de mala absorción. No suele encontrarse eosinofilia y, en ocasiones, se asocia a un déficit de disacaridasas con intolerancia a la lactosa.

Cabe mencionar que, el diagnóstico se realiza mediante el examen microscópico en fresco de heces. En él, las heces deben analizarse en la hora siguiente a la expulsión o conservarse con alcohol polivinílico o formol al 10\%. Pueden darse falsos negativos en el inicio de la enfermedad. En casos de fuerte sospecha y con varios estudios de heces en fresco negativos, se pueden hacer un aspirado y biopsia duodenal (método más sensible pero costoso e invasivo). Esta prueba sólo se hace en centros en los que se realizan endoscopias de rutina. Existen otros métodos diagnósticos, como enterotest (para estudio de trofozoítos en el jugo duodenal), ELISA (antígeno específico GSA-65) con una sensibilidad del 90-99\% y una especificidad del 95-100\%, PCR e inmuno fluorescencia con anticuerpos policlonales. Las pruebas serológicas para la detección de anticuerpos en el paciente, dada su escasa sensibilidad, sólo se recomiendan para estudios epidemiológicos.

\section{Enterobius Vermicularis.}

Este parásito, también llamado oxiuro, es el nematodo más común en nuestro país y se encuentra principalmente en población infantil. Es rara la infección en lactantes y niños pequeños, afecta principalmente entre los5 y 14 años. Se transmite por vía fecal-oral, auto infestación, ingestión de alimentos o tierra contaminada o inhalación de polvo de las casas. También, puede transmitirse a través de la ropa interior y de cama, objetos, etc. Los huevos contaminan extensas áreas del hogar, permaneciendo durante semanas en ropa, suelo y uñas. Ello exige una serie de medidas higiénicas además del tratamiento médico.

Probablemente, los niños que practican la onicofagia tienen mayor probabilidad de re infestarse, y este hábito es frecuente entre los parasitados por E. vermicularis. Desde la ingesta de los huevos a su excreción, nuevamente transcurren 2-3 semanas, lo que justifica repetir el tratamiento 


\section{Tratamiento de parasitosis intestinal en niños menores de 5 años}

Vol. 3, núm. 1., (2019)

Ronald Alfredo Cevallos Macías; Diana Katherine Suárez Intriago; Silvia Karina Briones

Valencia, Evelyn Estefanía Calderón López; Marvin Damián Veliz Mero; Marcos Andrés Campozano Burgos

antihelmíntico a los 15días; ya que, el tratamiento es efectivo frente a los gusanos que habitan la región ileocecal pero no frente a los huevos.

Es importante conocer que, en algunos de los casos la infección puede ser asintomática o sintomática, o puede cursar con prurito anal o perianal (de gran intensidad, predominio vespertino y con frecuentes lesiones de rascado perianal); dolores abdominales en fosa ilíaca derecha; alteraciones del tránsito intestinal (diarrea, vómitos, heces mucosas); anorexia; irritabilidad, cambios de carácter tipo inestabilidad o agresividad; alteraciones del sueño terrore nocturnos (relacionados con el prurito); bruxismo o vaginitis y leucorrea en las niñas relacionados con el rascado.

En este tipo de enfermedad, el diagnóstico de laboratorio se realiza mediante el test de Graham (prueba de la cinta adhesiva) o bien la técnica de Markey (con torundas vaselinizadas). Se debe descartar siempre la co - infección por Dientamoeba fragilis, porque con frecuencia es transportada en la cáscara de los huevos de Enterobius vermicularis

\section{Cryptosporidium Parvum.}

Es un protozoo que se multiplica en las vellosidades del tubo digestivo. Se desconoce el mecanismo específico por el que produce la diarrea. Se sitúa en el borde en cepillo de las células epiteliales sin invadirlas. La criptosporidiosis es una zoonosis que se transmite por vía fecal-oral, persona a persona o bien por la ingestión de agua (los ooquistes son resistentes a la cloración) o alimentos contaminados con ooquistes o contacto estrecho con animales infectados (ganado vacuno). Su prevención, por tanto, pasa por extremar las medidas higiénicas que eviten la transmisión interpersonal. Esta vía parece ser la responsable de la infección de niños que asisten a guarderías y de la alta prevalencia, en general, en preescolares. Los familiares de niños afectados se infectan en una proporción del 70\%. Existen tres formas clínicas:

- Forma asintomática: es frecuente en guarderías. Se produce una eliminación prolongada de ooquistes. 


\section{Tratamiento de parasitosis intestinal en niños menores de 5 años}

Vol. 3, núm. 1., (2019)

Ronald Alfredo Cevallos Macías; Diana Katherine Suárez Intriago; Silvia Karina Briones Valencia, Evelyn Estefanía Calderón López; Marvin Damián Veliz Mero; Marcos Andrés

Campozano Burgos

- Forma intestinal: gastroenteritis auto limitadas, con deposiciones acuosas sin sangre, dolor abdominal cólico, anorexia, vómitos y fiebre en el 30-50\% de los pacientes. En ocasiones, puede provocar síntomas inespecíficos como mialgias, debilidad y cefalea. Dura aproximadamente14 días sin tratamiento. La expulsión de ooquistes se mantiene durante varias semanas después de la desaparición de la diarrea.

- Forma extraintestinal (más frecuente en inmunodeprimidos): hepatitis, colecistitis, artritis reactivas y síntomas respiratorios

Blastocystis hominis,

Protozoo habitante del tracto gastrointestinal humano y considerado durante mucho tiempo como comensal no patógeno. Publicaciones recientes relacionan determinadas cepas del parásito con diversos síntomas, tanto intestinales (diarrea aguda auto limitada), como extra intestinales (alérgicos principalmente). Muchos autores aconsejan el tratamiento cuando se detecta en cantidades importantes en tres muestras consecutivas de heces, y sin que exista otro organismo potencialmente responsable de la clínica. La infección por Blastocystis cursa con malestar, anorexia y distensión abdominal, cólico, diarrea, flato y estreñimiento que alterna con diarrea. Menos frecuentemente, encontramos náuseas, vómitos, mareos, pérdida de peso, prurito y tenesmo. Se puede asociar con otros parásitos. Se diagnostica mediante el estudio microscópico de las heces

\section{Entamoeba histolytica.}

Actualmente, se conocen dos especies distintas de Entamoeba histolytica morfológicamente indistinguibles que parasitan al hombre: E. histolytica, la especie patógena e invasiva, y E. dispar, la especie de mayor prevalencia (diez veces superior a la anterior), comensal intestinal no patógeno y asociada a un estado de portador asintomático. Otras cinco especies no patógenas pueden colonizar, en raras ocasiones, el aparato intestinal humano: E. coli, E. hartmanni, E. gingivalis, E. moshkovskii y E.polecki. Se produce por la ingestión de los quistes parasitarios (a través del agua, de alimentos contaminados o contacto fecal-oral), resistentes a las concentraciones de cloro que se 


\section{Tratamiento de parasitosis intestinal en niños menores de 5 años}

Vol. 3, núm. 1., (2019)

Ronald Alfredo Cevallos Macías; Diana Katherine Suárez Intriago; Silvia Karina Briones

Valencia, Evelyn Estefanía Calderón López; Marvin Damián Veliz Mero; Marcos Andrés

Campozano Burgos

utilizan habitualmente en la potabilización de las aguas, pero muy sensibles al calentamiento hasta 55 grados.

Este tipo de parásitos, se diferencian tres formas parasitarias: trofozoíto (forma invasiva), prequiste y quiste. Los trofozoítos ameboides se adhieren en el colon (a nivel de ciego, colon ascendente y rectosigma), dando lugar a úlceras. En las invasiones más profundas pasan al sistema portal y al hígado. El $90 \%$ de los sujetos infectados por E. histolytica están asintomáticos y sólo en un 2-8\% se produce la invasión. En este último caso, se pueden presentar tres formas clínicas:

- Diarrea acuosa crónica (90\%): se produce colitis no disentérica con diarrea intermitente con moco sin sangre, dolor abdominal y flatulencia, y alternancia con estreñimiento.

- Disentería amebiana (10\%): de comienzo gradual, de posiciones intermitentes con sangre y moco, fiebre en sólo un 30\% de los casos, asociada a "pujo" (necesidad de defecar con mucho esfuerzo, pero cada vez menos material fecal y más sangre y moco) y tenesmo (espasmo doloroso en el recto que produce la necesidad de una nueva defecación) que se prolonga durante 1-2 semanas. Es característica la ausencia de síntomas consuntivos generales. En lactantes y niños pequeños de países tropicales o pacientes con SIDA, podemos encontrar colitis amebiana necrotizante fulminante.

- Forma crónica: es muy rara, pues cursa con brotes de dolor abdominal y diarrea sanguinolenta que se mantienen a lo largo de los años y pueden simular una enfermedad inflamatoria intestinal. Se diagnostica mediante el examen microscópico de heces (el estudio de tres muestras de heces frescas tiene una sensibilidad del 90\%; las muestras de heces frescas deben analizarse en los 30 minutos de su expulsión), serología con detección de anticuerpos antiamebianos (resultados positivos en un 95\% de pacientes con enfermedad sintomática de más de 7 días de duración y en la mayoría de portadores asintomáticos de E. histolytica, porque la E. dispar no despierta respuesta humoral), biopsia intestinal (se pueden encontrar trofozoítos móviles), detección de antígeno amebiano en heces o suero mediante contra inmuno lectroforesis o ELISA y la técnica de PCR (permiten distinguir entre las dos especies de Entamoeba). La infección por E. histolytica, aunque 


\section{Tratamiento de parasitosis intestinal en niños menores de 5 años}

Vol. 3, núm. 1., (2019)

Ronald Alfredo Cevallos Macías; Diana Katherine Suárez Intriago; Silvia Karina Briones Valencia, Evelyn Estefanía Calderón López; Marvin Damián Veliz Mero; Marcos Andrés

Campozano Burgos

sea asintomática, debe ser tratada porque puede hacerse invasora. Se debe hacer un control a las 2 - 4semanas del tratamiento y repetir hasta que el resultado sea negativo

\section{Trichuris trichiura.}

El hombre se infesta al ingerir por vía oral (a través de las manos, alimentos o bebidas contaminadas) huevos larvados del helminto. Tras la ingesta, el huevo eclosiona en el intestino delgado, migrando posteriormente al intestino grueso, donde madura hasta alcanzar el estado adulto. Se localiza, definitivamente, en el ciego. La mayoría de pacientes infestados por tricocéfalos son asintomáticos. En los casos sintomáticos, se producen vagas molestias abdominales, cólicos y distensión. En los casos graves, se puede encontrar diarrea sanguinolenta, prolapso rectal y anemia

\section{Ascaris lumbricoides.}

Es el nematodo más grande (puede medir hasta $35 \mathrm{~cm}$ ). Se transmite a través del suelo (donde pueden sobrevivir durante meses). La defecación indiscriminada y el uso de estiércol humano son la causa más importante de endemia por este parásito. El modo de transmisión al hombre es fecaloral, con dedos contaminados por contacto con el suelo. Los alimentos pueden contaminarse por las moscas o los fertilizantes. Tras la ingesta delos huevos, las larvas se liberan en el intestino, atraviesan la pared intestinal y, a través de la circulación venosa, migran a los pulmones. Posteriormente, se vuelven a deglutir localizándose la forma adulta en el intestino delgado (yeyuno e íleon).

En general, esta infección suele ser asintomática y raras veces conducir a complicaciones graves. Cuando es sintomática, se manifiesta por dos fases distintas desde el punto de vista clínico y diagnóstico: la fase de la migración larvaria pulmonar y la fase digestiva producida por los gusanos adultos.

- Fase larvaria: causa una neumonitis pulmonar (síndrome de Löeffler) con tos espasmódica, expectoración mucosa, fiebre elevada y hemoptisis ocasional. A veces 


\section{Tratamiento de parasitosis intestinal en niños menores de 5 años}

Vol. 3, núm. 1., (2019)

Ronald Alfredo Cevallos Macías; Diana Katherine Suárez Intriago; Silvia Karina Briones

Valencia, Evelyn Estefanía Calderón López; Marvin Damián Veliz Mero; Marcos Andrés

Campozano Burgos

urticaria y angioedema. Las lesiones pulmonares se producen por la migración de las larvas al alvéolo, con la reaccióninflamatoria correspondiente.

- Fase digestiva: cursa con dolor abdominal cólico en región epigástrica, náuseas matutinas, vómitos y, a veces, diarrea. En la infancia, puede aparecer detención del desarrollo pondero estatural. Debido a su tamaño puede producir: obstrucción intestinal, pancreatitis, colecistitis, ictericia y absceso hepático. Para el diagnóstico, hay que demostrar la presencia de huevos. La presencia del parásito adulto en vómitos o en las heces. En la fase larvaria, pueden encontrarse larvas en el esputo, junto con una clínica y radiología sugestivas. Es frecuente la hiper eosinofília, con aumento progresivo hasta la tercera semana de la infección y, posteriormente, una disminución paulatina hasta la emisión de huevos. Se debe hacer un control tras 2-4 semanas del tratamiento.

Taenia solium o Taenia saginata.

Los huéspedes intermediarios de estos cestodos son los cerdos (T. solium) y bóvidos (T. saginata). Los humanos se infestan al ingerir carne cruda o poco cocinada. La cocción completa o la refrigeración destruyen al parásito. La mayoría de los casos son asintomáticos (se detecta al darse cuenta el paciente, que elimina proglótides [anillos] del (parásito). Los casos sintomáticos cursan con dolor epigástrico, anorexia o aumento del apetito, dispepsia, náuseas o vómitos e insomnio. En ocasiones, se producen alteraciones neurológicas, como: cefalea, vértigos o nerviosismo. Se diagnostican por detección de huevos en el examen microscópico delas heces o bien la presencia de proglótides en las mismas. Es importante conocer que los anillos de T. saginata presentan movilidad. Es frecuente la hipereo sinofilia.

Hymenolepis nana o Hymenolepis diminuta.

La Hymenolepis nana o "tenia enana” constituye la cestodiasis humana más frecuente. La misma, afecta principalmente a niños, que pueden presentar parasitaciones múltiples con el consiguiente retraso en el desarrollo ponderal. La transmisión se produce por la vía mano-boca o a través de alimentos y bebidas contaminadas. En el caso de Hymenolepis diminuta, el hombre puede infestarse por la ingesta accidental de insectos parasitados presentes en cereales y harinas 


\section{Tratamiento de parasitosis intestinal en niños menores de 5 años}

Vol. 3, núm. 1., (2019)

Ronald Alfredo Cevallos Macías; Diana Katherine Suárez Intriago; Silvia Karina Briones Valencia, Evelyn Estefanía Calderón López; Marvin Damián Veliz Mero; Marcos Andrés

Campozano Burgos

Tratamiento preventivo de la Parasitosis Intestinal.

Tratamiento Clínico en base a medicina Convencional.

- En pacientes inmuno competentes, la curación es espontánea en 2 o 3 días. Hay que tener en cuenta el estado nutricional del paciente y las medidas de soporte de reposición de líquidos y electrolitos, si la diarrea es grave. $\mathrm{Si}$ decidimos tratar, especialmente en inmunodeprimidos, en el caso de los niños de 1 a 3 años, la elección es Nitazoxamida, a la dosis de $100 \mathrm{mg} / 12 \mathrm{~h}$, durante 3 días; mientras que, en los niños de 4 a 11 años, la dosis es doble, $200 \mathrm{mg} / 12 \mathrm{~h}$, también durante 3 días.

- El tratamiento amiento alternativo es la para momicina, a $25-35 \mathrm{mg} / \mathrm{kg} / \mathrm{dí} / \mathrm{a} / 8 \mathrm{~h}$, durante 10 días. En pacientes con SIDA, la terapia antirretroviral consigue mejoría en el estatus inmune y disminuir la sintomatología. Cuando el médico tratante se encuentra en presencia en presencia de anemia debe en primer lugar, tratar la misma. La prevención se basa en el uso de letrinas, calzado, saneamiento ambiental, medidas de educación a la población y tratamiento comunitario en zonas de alta endemia. De allí, que es primordial tratar a toda la familia y extremar las medidas de higiene; ya que, aproximadamente, el $40 \%$ de los niños en edad escolar están infectados.

- En la presencia de Enterobius vermicularis el tratamiento de elección es Mebendazol, $100 \mathrm{mg}$ dosis única, pero es conveniente repetir en dos semanas. La alternativa es el tratamiento con Pamoato de pyrantel, $11 \mathrm{mg} / \mathrm{kg}$ dosis única, máximo $1 \mathrm{~g}$. Repetir en 2 semanas.

- Si el caso es de Giardia lamblia, el tratamiento de elección (tanto si es asintomático como sintomático) es el metronidazol $15-30 \mathrm{mg} / \mathrm{kg} /$ día $/ 8 \mathrm{~h}$, durante $5-7$ días, repetir al cabo de una semana. El tratamiento alternativo es el tinidazol, $50 \mathrm{mg} / \mathrm{kg} / 24 \mathrm{~h}$ solo 1 día o mepacrina a $8 \mathrm{mg} / \mathrm{kg}$ cada $8 \mathrm{~h}$, durante 5 días. 


\section{Tratamiento de parasitosis intestinal en niños menores de 5 años}

Vol. 3, núm. 1., (2019)

Ronald Alfredo Cevallos Macías; Diana Katherine Suárez Intriago; Silvia Karina Briones

Valencia, Evelyn Estefanía Calderón López; Marvin Damián Veliz Mero; Marcos Andrés

Campozano Burgos

- Si se trata de Protozoos, El tratamiento de elección (tanto si es asintomático como sintomático) es el metronidazol 15-30 mg/kg/día/8 h, durante 5-7 días, repetir al cabo de una semana. El tratamiento alternativo es el tinidazol, $50 \mathrm{mg} / \mathrm{kg} / 24 \mathrm{~h}$ solo 1 día o mepacrina a $8 \mathrm{mg} / \mathrm{kg}$ cada $8 \mathrm{~h}$, durante 5 días. De persistir la clínica tras el tratamiento, volver a tratar y plantearse descartar inmunodeficiencia (sobre todo, déficit de $\operatorname{IgA}$ ) y volver a tratar con metronidazol, tinidazol (50-60 mg/kg/24 h, dosis única), o mepacrina $(8 \mathrm{mg} / \mathrm{kg} / 8 \mathrm{~h})$. Otra alternativa al tratamiento es el albendazol, $15 \mathrm{mg} / \mathrm{kg} / 24 \mathrm{~h}$, durante 5 días.

- Cuando se trata de Entamoeba histolytica, el tratamiento se basa en Amebicidas intra luminales: la absorción es baja, actúan a nivel intestinal y son activos frente a quistes y trofozoítos. En niños, la elección es la Para momicina, a 25-35 mg/kg/día/8 h, durante 7 días. Y la alternativa es el Iodoquinol, 30-40 mg/kg/día/8 h, durante 20 días. Amebicidas sistémicos: en niños, el tratamiento de elección es metronidazol, a 30-50 mg/kg/día/8 h, durante 10 días. El tratamiento alternativo es tinidazol, 50 mg/kg/día (máximo: $2 \mathrm{~g}$ ), de 3 a 10 días. En caso de absceso hepático y poca respuesta al tratamiento, se debe añadir cloroquina.

- Si el paciente padece de Criptosporidiasis, el tratamiento en pacientes inmuno competentes, la curación es espontánea en 2 o 3 días. Hay que tener en cuenta el estado nutricional del paciente y las medidas de soporte de reposición de líquidos y electrolitos, si la diarrea es grave. Si decidimos tratar, especialmente en inmunodeprimidos, en el caso de los niños de 1 a 3 años, la elección es Nitazoxamida, a la dosis de 100 mg/12 h, durante 3 días; mientras que, en los niños de 4 a 11 años, la dosis es doble, 200 mg/12 h, también durante 3 días.

- El tratamiento alternativo es la para momicina, a $25-35 \mathrm{mg} / \mathrm{kg} /$ día $/ 8$ h, durante 10 días. En pacientes con SIDA, la terapia antirretroviral consigue mejoría en el estatus inmune y disminuir la sintomatología.

- Si el médico tratante se encuentra con Helmintos, el tratamiento de elección es Mebendazol, $100 \mathrm{mg}$ dosis única, pero es conveniente repetir en dos semanas. La 


\section{Tratamiento de parasitosis intestinal en niños menores de 5 años}

Vol. 3, núm. 1., (2019)

Ronald Alfredo Cevallos Macías; Diana Katherine Suárez Intriago; Silvia Karina Briones Valencia, Evelyn Estefanía Calderón López; Marvin Damián Veliz Mero; Marcos Andrés

Campozano Burgos

alternativa es el tratamiento con Pamoato de pyrantel, $11 \mathrm{mg} / \mathrm{kg}$ dosis única, máximo $1 \mathrm{~g}$. Repetir en 2 semanas.

- Si el paciente presenta Trichuris trichiura, el tratamiento de elección es el mebendazol, 100 mg/12 h 3 días o 500 mg dosis única, o Albendazol, 200-400 mg/día, dosis única. Se han de extremar las medidas de higiene personal y la eliminación de heces ha de ser adecuada, utilización de agua potable y correcto lavado de alimentos. Y se ha de extremar la vigilancia de los juegos con tierra y arena de los niños en los parques y realizar adecuada higiene de manos.

- Si el caso presenta Ascaris lumbricoides, aplicarse mebendazol, 100 mg/12 h 3 días o $500 \mathrm{mg}$ dosis única, o Albendazol, 200-400 mg/día, dosis única de extremar las medidas de higiene personal y la eliminación de heces ha de ser adecuada, utilización de agua potable y correcto lavado de alimentos. Y se ha de extremar la vigilancia de los juegos con tierra y arena de los niños en los parques y realizar adecuada higiene de mano

- Si el tratante se encuentra con paciente con Ancylostoma duodenale y Necator americanus (Uncinarias) atamiento de elección es Mebendazol, 100 mg dosis única, pero es conveniente repetir en dos semanas. La alternativa es el tratamiento con Pamoato de pyrantel, $11 \mathrm{mg} / \mathrm{kg}$ dosis única, máximo $1 \mathrm{~g}$. Repetir en 2 semanas.

- Si la situación clínica del paciente presenta Strongyloides stercolaris El tratamiento de elección es la Ivermectina, 0,2 mg/kg/día cada 24 h, máximo $12 \mathrm{mg}$ y durante tres días. La alternativa es Albendazol, 200 mg/12 h 3-5 días y, si existe hiper infestación, el tratamiento ha de durar 7 días. En pacientes inmunodeprimidos y si existe hiper infestación, hace falta repetir el tratamiento una semana más tarde. La curación ha de ser comprobada por la ausencia de parásitos. La prevención se basa en el uso de letrinas, calzado, saneamiento ambiental y educación a la población.

- Cuando el caso presenta Hymenolepis, En las teniasis por T. solium o T. saginata, Praziquantel, a $10 \mathrm{mg} / \mathrm{kg} /$ día $/ 24 \mathrm{~h}$, solo 1 día, o Niclosamida a 50 mg/kg/día/24 h, 1 día. Cisticercosis: Albendazol 15-20 mg/kg/día/12 h, máximo: 800 


\section{Tratamiento de parasitosis intestinal en niños menores de 5 años}

Vol. 3, núm. 1., (2019)

Ronald Alfredo Cevallos Macías; Diana Katherine Suárez Intriago; Silvia Karina Briones Valencia, Evelyn Estefanía Calderón López; Marvin Damián Veliz Mero; Marcos Andrés Campozano Burgos

mg, 14-28 días, o Praziquantel, $50 \mathrm{mg} / \mathrm{kg} /$ día/8 h, durante 15 días. Se deben administrar junto a corticoides

- Si el caso presenta Fasciola hepática El tratamiento se basa en el triclabendazol, 10 $\mathrm{mg} / \mathrm{kg}$ en una o dos dosis por vía oral; en casos graves, se puede repetir a las $12 \mathrm{~h}$. La alternativa es Bithionol, 30-50 mg/kg oral a días alternos, de 10-15 dosis en total. En los casos graves, se deben administrar corticoides simultáneamente.

\section{Tratamiento Preventivo en base a Normas Sanitarias:}

- Higiene del Hogar: La higiene del hogar, representa una de las normas o medidas que toda persona y familia debe cumplir a fin de minimizar el riesgo de adquirir enfermedades como la parasitosis intestinal. Al respecto Guido (2011g), refiere "esta medida pretende cumplir en la eliminación de desechos del medio" (p.76), lo cual implica desechar las fuentes de infecciones que pudieran encontrarse en calles, aceras, entre otras, así, por ejemplo, que las personas dentro de sus casas actúen en función de eliminar basura en el cual sea de utilidad para todos los habitantes.

- Esto dará como resultado disminuir fuente para el criadero de los parásitos en niños en esta parte es de gran relevancia que las madres se les oriente de manera teórica - práctica, como debe ser eliminada la basura del hogar, los pañales usados, residuos de alimentos, así como también limpieza de pipotes y bolsas de basuras. Por consiguiente, la higiene del hogar puede ser ilustrada con imágenes que proyecten el bienestar y confort de la familia, lo cual se refiere a la búsqueda de acciones apropiadas para implementar la eliminación de roedores, lavados de las sabanas y cortinas, aseo de mesas y sillas, barrido de patio y frente, lavado de baño.

- Servicio de Agua. Desde tiempos remotos, se ha hablado acerca del consumo de agua potable en el requerimiento saludable del ser humano como uno de los cuidados básicos para la salud. En ese sentido, se hace necesario que a las madres capacitadas se les proporcione información como a los demás participantes, además del beneficio del consumo de agua potable las medidas necesarias para contar con la misma que incluye su 


\section{Tratamiento de parasitosis intestinal en niños menores de 5 años}

Vol. 3, núm. 1., (2019)

Ronald Alfredo Cevallos Macías; Diana Katherine Suárez Intriago; Silvia Karina Briones Valencia, Evelyn Estefanía Calderón López; Marvin Damián Veliz Mero; Marcos Andrés

Campozano Burgos

hervido y conservación en recipientes adecuados, así como también, estrategias que faciliten un transporte adecuado de agua para el consumo.

- Estas estrategias están basadas en el principio que puedan alcanzar que se les aporte la disposición de agua por intermedio de tuberías y no por camiones cisternas en el cual su desplazamiento en oportunidades es disperso. Estas acciones permiten interpretar que las madres capacitadas además de fomentar acciones de Educación para la Salud, también proporcionen la información necesaria para que comprendan la importancia del uso de agua tratada dentro de un marco operacional, intersectorial e interinstitucional en la búsqueda de mejoras para la producción y provisión de servicios para un consumo de alimentos que garantice la prevención de parasitosis en el niño.

- Lavado de Manos: Esta norma representa una de las medidas preventivas de más conocimiento social. La misma, es considerada por Pérez (2012), quien plantea que, el "lavado de las manos con agua y jabón antes y después de preparar los alimentos, así como también tomar las medidas pertinentes antes y después de ir al baño" (p.16). Este conocimiento, es de vital importancia, el mismo permitirá a las madres que conozcan que se deben adoptar ciertas prácticas de higiene para ayudar a proteger de las parasitosis a los niños, entre ellas el lavado de las manos, el cual puede diferir de una cultura a otra.

- Lavarse bien las manos significa usar jabón o un sustituto, usar bastante agua y limpiarse cuidadosamente completamente las manos, por lo tanto todos los miembros de la familia deben lavarse bien las manos, después de limpiar y desechar las heces de un niño que haya defecado, después de defecar, antes de preparar los alimentos, antes de comer y lavarle las manos al niño antes de alimentarlo. El perfeccionamiento del aporte de conocimiento del lavado de manos tiene implicación para la protección del transporte de microorganismos coadyuvantes a la aparición de las parasitosis.

- Higiene de los Alimentos: Este hábito y norma social, debe realizarse antes y después de preparar los alimentos, manteniéndolos tapados contribuirá de esta forma al mantenimiento del cuidado, refiere Pérez ( $\mathrm{Ob}$ cit), que este tipo de conducta preventiva "ha sido considerada como una de las estrategias básicas que están impuestas por las propias 


\section{Tratamiento de parasitosis intestinal en niños menores de 5 años}

Vol. 3, núm. 1., (2019)

Ronald Alfredo Cevallos Macías; Diana Katherine Suárez Intriago; Silvia Karina Briones

Valencia, Evelyn Estefanía Calderón López; Marvin Damián Veliz Mero; Marcos Andrés Campozano Burgos

personas" (p.30). He aquí, que el cumplimiento de esta conducta significa el entendimiento lógico y necesario de cumplir con las medidas pertinentes para el manejo efectivo en el cuidado del niño menor de 5 años.

- Lavado de Frutas: El lavado de frutas, hortalizas y legumbres con agua antes de ser consumidos y hervidos aquellos que son requeridos, también son acciones necesarias a tener en cuenta durante el aporte informativo a la capacitación de las madres, esto es a manera de proyectar dentro de los participantes el beneficio que genera la práctica higiénica saludable. En esta parte es necesario insistir en el lavado de hortalizas y frutas, previamente antes de ser consumidas por el niño.

\section{Conclusiones.}

Las parasitosis intestinales son infecciones del tubo digestivo, que pueden producirse por la ingestión de quistes de protozoos, huevos o larvas de gusanos, o por la penetración de larvas por vía transcutánea, desde el suelo. Cada parásito va a realizar un recorrido específico en el huésped y afectará a uno o varios órganos, según sea este recorrido. Estas infecciones se pueden clasificar según el tipo de parásito y la afectación que provoquen en los diferentes órganos y sistemas. También, es importante saber reconocer algunas especies que no requieren tratamiento porque no son patógenas para los humanos.

La importancia de conocer sobre estos parásitos intestinales radica en que representan una de las problemáticas de salud de mayor incidencia a nivel mundial, pues infectan a más de la mitad de la población humana siendo la población pediátrica es la más afectada. Las consecuencias no son del todo conocidas ni valoradas en toda su profundidad, ya que presentan una elevada morbilidad y mortalidad, sobre todo, en países sub desarrollados.

Estos parásitos, pueden causar patología grave e incluso causar mortalidad. En cuanto al tratamiento, hay pocas novedades, sigue siendo farmacológico. En ocasiones, son medicamentos extranjeros que hay que solicitar específicamente, e individualmente para cada caso, y no se tienen almacenados. Es importante, por tanto, además de conocer las dosis y como realizar la 


\section{Tratamiento de parasitosis intestinal en niños menores de 5 años}

Vol. 3, núm. 1., (2019)

Ronald Alfredo Cevallos Macías; Diana Katherine Suárez Intriago; Silvia Karina Briones Valencia, Evelyn Estefanía Calderón López; Marvin Damián Veliz Mero; Marcos Andrés

Campozano Burgos

administración de los fármacos, saber cómo conseguirlos, siguiendo el procedimiento de solicitud de medicación extranjera.

La infección intestinal generada a causa de parasitosis intestinal produce en el afectado una serie de signos y síntomas. Su tratamiento, va a depender de la naturaleza del agente causal, de la duración de su acción, y de la resistencia del paciente y del grado de afectación, generando sobre todo en la población infantil una serie de complicaciones entre las que se pueden mencionar, la deshidratación; siendo ésta la primera alteración, la cual produce pocas variaciones en la composición electrolítica o ácido-base, por otra parte, se encuentran los desequilibrios en su medio interno, lo que hace indispensable su pronta corrección a fin de evitar riesgos y complicaciones.

\section{Bibliografía.}

Arias, F. (2010). Paradigmas de la Investigación Científica. España: Editorial: Luces.

Blanco, A. (2010). Parasitosis en niños menores de Cinco Años de Edad. Perú: Trabajo de grado no publicado realizado en la Universidad Católica Santo Toribio de Mogrovejo en Chiclayo Perú.

Blanco, U. (2011). Epidemiología de la Parasitosis Intestinal. España: Editorial: Andaluz.

Dávila, N. (2012). Paradigmas de la Investigación Científica. Pereire, Colombia: Editorial: Las Brisas.

León, S. (2011). Parasitosis Intestinal en niños menores de Cinco Años. Boletín médico de Posgrado de la Facultad de Medicina de la Universidad del Zulia., 21.

OMS, O. M. (2017). Epidemiología de la Parasitosis Intestinal. Revista Salud Integral de la Escuela de Medicina de la Universidad de la Habana Cuba, 13.

OPS, O. P. (2011). Enfermedades Parasitarias en Niños. Boletín médico de Posgrado de la Facultad de Medicina de la Universidad del Zulia., 12. 


\section{Tratamiento de parasitosis intestinal en niños menores de 5 años}

Vol. 3, núm. 1., (2019)

Ronald Alfredo Cevallos Macías; Diana Katherine Suárez Intriago; Silvia Karina Briones

Valencia, Evelyn Estefanía Calderón López; Marvin Damián Veliz Mero; Marcos Andrés

Campozano Burgos

Rossiet, H. (2011). Enfermedades Parasitarias. Artículo del Periódico EL IMPULSO. Sección Salud. Lara - Venezuela, 32.

Vianco, C. (2011). Parasitosis Intestinal, Causas y Consecuencias. Revista de Trabajo Social, Universidad de Huelva, 32.

Vieira, H. (2011). Parasitosis Intestinal. Infección Gástrica de gran incidencia. Bogotá: Editorial Limusa. 